\title{
Relation between microRNA expression in peritoneal dialysis effluent and peritoneal transport characteristics
}

\author{
Jin Chen ${ }^{\mathrm{a}, \mathrm{b}}$, Philip Kam-Tao ${ }^{\mathrm{a}}$, Bonnie Ching-Ha Kwan ${ }^{\mathrm{a}}$, Kai-Ming Chow ${ }^{\mathrm{a}}$, Ka-Bik Lai ${ }^{\mathrm{a}}$, \\ Cathy Choi-Wan Luk ${ }^{\mathrm{a}}$ and Cheuk-Chun Szeto ${ }^{\mathrm{a}, *}$ \\ ${ }^{a}$ Department of Medicine and Therapeutics, Prince of Wales Hospital, The Chinese University of Hong Kong, \\ Shatin, Hong Kong, China \\ ${ }^{\mathrm{b}}$ Division of Nephrology, Sichuan Academy of Medical Sciences and Sichuan Provincial People's Hospital, \\ Chengdu, Sichuan, China
}

\begin{abstract}
Background: The role of microRNAs (miRNAs) in peritoneal transport is uncertain.
Methods: We studied 82 new peritoneal dialysis (PD) patients, 22 prevalent patients without ultrafiltration problem, and 6 patients with documented ultrafiltration problem. Peritoneal transport was determined by standard peritoneal equilibration test (PET). RNA was extracted from the PD effluent after PET, and intra-peritoneal expression of miRNA targets were quantified.

Results: There were significant difference in the PDE expressions of miR-15a and miR-21. There were modest inverse correlations between ultrafiltration volume and PDE expression of miR-17 $(r=-0.198, p=0.041)$ and miR-377 $(r=-0.201, p=$ 0.041). There was an inverse correlations between dialysate-to-plasma creatinine concentration at 4 hours and PDE expression of miR-192 ( $r=-0.199, p=0.040)$; while mass transfer area coefficient of creatinine correlated with PDE expression of miR-192 $(r=-0.191, p=0.049)$ and miR-377 $(r=0.201, p=0.041)$. Amongst 7 randomly selected patients who had repeat PET after one year, there was a significant correlation between baseline PDE expression of miR-377 and change in ultrafiltration volume $(r=-0.852, p=0.015)$.

Conclusion: The miRNA expression in PDE, including miR-15a, miR-17, miR-21, miR-30, miR-192, and miR-377, correlated with peritoneal transport characteristics. Our result suggests that miRNA may play a role in the regulation of peritoneal membrane function.
\end{abstract}

Keywords: Gene expression, chronic renal failure, peritoneal equilibration test

\section{Introduction}

Peritoneal dialysis (PD) is the first-line treatment of end stage kidney disease in Hong Kong [1]. The success of PD depends on the sustained ability of the peritoneum to act as a semi-permeable membrane [2], and peritoneal failure is the major cause of technique failure in PD patients.

Peritoneal failure is characterized by new vessel formation, loss of mesothelial surface area, and depo-

\footnotetext{
${ }^{*}$ Corresponding author: Dr. C.C. Szeto, Department of Medicine and Therapeutics, Prince of Wales Hospital, The Chinese University of Hong Kong, Shatin, NT, Hong Kong, China. Tel.: +852 2632 3126; Fax: +852 2637 3852; E-mail: ccszeto@cuhk.edu.hk.
}

sition of extracellular matrix. Peritoneal mesothelial cell (PMC) is important in the homeostasis of the peritoneal membrane, and plays active roles in the synthesis and remodeling of extra-cellular matrix [3]. Previous studies show that PMC undergoes transdifferentiation from epithelial to fibroblast-like phenotype after prolonged PD [4,5]. In fact, soon after dialysis is initiated, PMC undergo epithelial-mesenchymal transition (EMT), with a progressive loss of epithelial morphology [5]. However, the detailed mechanism of mesothelial cell EMT is incompletely understood.

Recently, the importance of microRNAs (miRNAs) in the process of EMT is being recognized. MicroRNAs are a family of 21- to 25-nucleotide, non-coding small RNAs that primarily function as gene regula- 
tors $[6,7]$ by interacting with multiple mRNAs and inducing either translation suppression or degradation of mRNA [6,7]. Previous experiments showed that miRNAs are involved in the regulation of cell-cycle progression, apoptosis, DNA repair, and angiogenesis in many organ systems [8-10]. Recent data suggest a pivotal role of miRNA in the regulation of epithelial EMT. For example, in murine epithelial cell models of TGF- $\beta$-induced EMT, members of the miR-200 family and miR-205 were repressed during EMT, while over-expression of this family hindered EMT by enhancing E-cadherin expression through direct targeting of zinc-finger E-box binding homeobox 1 (ZEB1) and ZEB2, which encode transcriptional repressors of Ecadherin [11-13]. However, the role of miRNA in peritoneal transport or EMT of peritoneal mesothelial cells is not well understood. In this study, we examined the relationship between miRNA expression in PD effluent and clinical peritoneal transport in peritoneal dialysis patients.

\section{Patients and methods}

\subsection{Overall arrangement}

We studied 82 consecutive new PD patients in our unit from May 2009 to December 2010. Patients who are unlikely to survive for 6 months, who are planned to have elective living donor transplant or transfer to other renal center within 6 months will be excluded. As part of the regular medical care, all patients required standard peritoneal equilibration test (PET) and dialysis adequacy assessment around 4 weeks after they were stable on PD. In addition, we studied 28 prevalent PD patients who had clinical problem of ultrafiltration and needed PET (either suspected peritoneal failure because of recurrent hospital admission for fluid overload or a history of recurrent peritonitis). Patients with recent peritonitis were studied at least 4 weeks after completion of antibiotics. After written informed consent, we collected an extra $50 \mathrm{ml}$ of peritoneal dialysis effluent (PDE) sample for miRNA study at the end of the PET. This study was approved by the Clinical Research Ethics Committee of our university.

\subsection{Peritoneal equilibration test (PET)}

We used the standard PET as described by Twardowski [14]. All patients were in euvolemic state during PET. Drainage and ultrafiltration volumes (UF) at
4 hour were documented. Dialysate-to-plasma ratios of creatinine (D/P) at 0,2 , and 4 hours were calculated after correction of glucose interference [15]. Mass transfer area coefficients of creatinine (MTAC) normalized for body surface area (BSA) was calculated by the formula described by Krediet [16]. The results of PET were plotted on a PET graph, and patients were classified into fast, fast-average, slow-average and slow transporter [14]. In addition, we measured the dialysate total protein level as a marker of peritoneal transport for large molecules [17].

\section{3. miRNA extraction from $P D E$}

The methods of RNA extraction from body fluid have been described previously [18]. Briefly, PDE specimen was collected and sent to laboratory for processing immediately or stored in $4{ }^{\circ} \mathrm{C}$ overnight. PDE sample was centrifuged at $3000-\mathrm{g}$ for 30 minutes and at 13000 - $\mathrm{g}$ for 5 minutes at $4{ }^{\circ} \mathrm{C}$. The centrifuge sediment was lysed by RNA lysis buffer (Qiagen Inc, Ontario, Canada). Specimens were then stored at $-80^{\circ} \mathrm{C}$ until use. MirVana ${ }^{\mathrm{TM}}$ miRNA isolation kit (Ambion, Inc. Austin, TX, USA) was used for the extraction of total RNA from PDE sediment according to the manufacturer's protocol. Our previous data showed that the integrity of RNA isolated from body fluid by this method was adequate for real time quantitative polymerase chain reaction (RT-QPCR) [19].

\section{4. $R T-Q P C R$}

Based on previous studies on the potential miRNA species that contribute to the process of EMT [20], we studied the following miRNA targets: miR-15a, miR17, miR-17-92, miR-21, miR-30, miR-192, miR-216a, miR-217, and miR-377. PDE expression of these specific microRNA species was quantified by RT-QPCR using the ABI Prism 7900 Sequence Detection System (Applied Biosystems, Foster City, CA, USA). Commercially available Taqman primers and probes, including 2 unlabeled PCR primers and $1 \mathrm{FAM}^{\mathrm{TM}}$ dyelabeled TaqMan ${ }^{\circledR}$ MGB probe were used for all the targets (all from Applied Biosystems). RNU48 (Applied Biosystems) was used as house-keeping genes to normalize the microRNA expression. Results were analyzed with Sequence Detection Software version 2.0 (Applied Biosystems). The $\Delta \Delta \mathrm{C}_{T}$ method for relative quantitation was used.

\subsection{Statistical analysis}

Statistical analysis was performed by SPSS version 15.0 software. Results were expressed as mean \pm SD 
Table 1

Baseline clinical characteristics

\begin{tabular}{lcccl}
\hline Group & New case & Prevalent case & UF fail case & P value \\
\hline No. of patients & 82 & 22 & 6 & \\
Sex (M:F) & $49: 33$ & $14: 8$ & $4: 2$ & $\mathrm{p}=0.9$ \\
Age (year) & $57.4 \pm 13.5$ & $55.8 \pm 13.8$ & $61.7 \pm 15.6$ & $p=0.6$ \\
Duration of dialysis (months) & $2.4 \pm 0.8$ & $17.3 \pm 20.0$ & $3.8 \pm 1.5$ & $p<0.0001$ \\
Renal diagnosis, no. of case (\%) & & & & $p=0.6$ \\
$\quad$ Glomerulonephritis & $24(29.3 \%)$ & $4(18.2 \%)$ & $3(50.0 \%)$ & \\
$\quad$ Diabetic nephropathy & $29(35.4 \%)$ & $13(59.1 \%)$ & $3(50.0 \%)$ & \\
Polycystic kidney & $7(8.5 \%)$ & 0 & 0 & \\
$\quad$ Hypertensive nephrosclerosis & $7(8.5 \%)$ & $1(4.5 \%)$ & 0 & \\
$\quad$ Obstructive uropathy & $4(4.9 \%)$ & $1(4.5 \%)$ & 0 & \\
$\quad$ Others / unknown & $11(13.5 \%)$ & $1(4.5 \%)$ & 0 & \\
Major comorbidity, no. of case (\%) & & & & \\
$\quad$ Diabetes & $32(39.0 \%)$ & $14(63.6 \%)$ & $4(66.7 \%)$ & $p=0.068$ \\
$\quad$ Ischemic heart disease & $15(18.3 \%)$ & $7(31.8 \%)$ & $2(33.3 \%)$ & $p=0.3$ \\
$\quad$ Cerebrovascular accident & $12(14.6 \%)$ & $7(31.8 \%)$ & $4(66.7 \%)$ & $p=0.004$ \\
Charlson's comorbidity score & $4.9 \pm 2.1$ & $5.7 \pm 3.0$ & $6.5 \pm 2.6$ & $p=0.12$ \\
\hline
\end{tabular}

Table 2

Baseline biochemical profile, peritoneal transport, and dialysis adequacy indices of the patients

\begin{tabular}{lcccl}
\hline & New case & Prevalent case & UF fail case & P value \\
\hline No. of patients & 82 & 22 & 6 & \\
Hemoglobin $(\mathrm{g} / \mathrm{dL})$ & $9.1 \pm 1.4$ & $8.5 \pm 1.9$ & $10.6 \pm 2.4$ & $p=0.061$ \\
Serum albumin (g/L) & $35.7 \pm 3.8$ & $35.4 \pm 5.5$ & $32.5 \pm 4.1$ & $p=0.4$ \\
Peritoneal transport & & & & \\
$\quad$ ultrafiltration (L) & $0.31 \pm 0.24$ & $0.35 \pm 0.26$ & $0.12 \pm 0.20$ & $p<0.0001$ \\
$\quad$ D/P creatinine & $0.586 \pm 0.122$ & $0.593 \pm 0.116$ & $0.689 \pm 0.121$ & $p<0.0001$ \\
$\quad$ MTAC creatinine & $7.82 \pm 3.41$ & $7.99 \pm 3.74$ & $9.76 \pm 3.33$ & $p=0.009$ \\
$\quad$ & & & \\
$\quad$ dialysate protein $(\mathrm{g} / \mathrm{L})$ & $1.00 \pm 0.41$ & $0.74 \pm 0.25$ & $0.99 \pm 0.33$ & $p=0.053$ \\
Total Kt/V & $2.18 \pm 0.50$ & $1.99 \pm 0.52$ & $2.25 \pm 0.40$ & $p=0.4$ \\
residual GFR (ml/min/1.73 $\left.\mathrm{m}^{2}\right)$ & $4.00 \pm 2.78$ & $1.78 \pm 2.00$ & $4.26 \pm 3.83$ & $p=0.015$ \\
NPNA $(\mathrm{g} / \mathrm{kg} / \mathrm{day})$ & $1.12 \pm 0.27$ & $1.25 \pm 0.20$ & $0.97 \pm 0.31$ & $p=0.092$ \\
\hline
\end{tabular}

D/P, dialysate-to-plasma concentration ratio of creatinine; MTAC, mass transfer area coefficient; GFR, glomerular filtration rate; NPNA, normalized protein nitrogen appearance.

for normally distributed data and median and range for skewed data. Since the gene expression data were highly skewed, data between group were compared by Mann Whitey U test, subgroup were compared by ANOVA, and comparisons between gene expression in PDE and peritoneal transport parameters were performed by the Spearman's partial correlation coefficient. All P-values were corrected for multiple comparison by the Bonferroni method. P-value below 0.05 was considered statistically significant. All probabilities were two-tailed.

\section{Results}

We studied 110 PD patients; 82 were new and 28 prevalent PD patients. Amongst the 28 prevalent patients, 6 had documented ultrafiltration problem by PET (UF fail group), while the other 22 had no objective ultrafiltration problem (prevalent group). The de- mographic and baseline clinical information are summarized in Table 1. Their baseline biochemical, peritoneal transport, and dialysis adequacy parameters are compared and summarized in Table 2. In short, most of the baseline clinical and biochemical parameters were highly comparable between the groups, except the prevalent cases had been on PD longer and had a lower residual GFR. In addition, diabetes and pre-existing cerebrovascular disease were more common in prevalent and UF failure cases. As expected, the UF failure cases had higher D/P at 4 hours and MTAC creatinine, as well as lower ultrafiltration volume than the other groups.

\subsection{Difference between groups}

We attempted to measure the expression of miR15a, miR-17, miR-21, miR-30, miR-216a, miR-217 and miR-377 in PDE. However, no detectable expres- 
Table 3

Internal correlations between PDE expression of miRNA targets

\begin{tabular}{llllll}
\hline & miR-17 & miR-21 & miR-30 & miR-192 & miR-377 \\
\hline miR-15a & $r=0.773, p<0.0001$ & $r=0.879, p<0.0001$ & $r=0.669, p<0.0001$ & $r=0.849, p<0.0001$ & $r=0.352, p=0.0003$ \\
miR-17 & & $r=0.809, p<0.0001$ & $r=0.686, p<0.0001$ & $r=0.712, p<0.0001$ & $r=0.346, p=0.0003$ \\
miR-21 & & & $r=0.723, p<0.0001$ & $r=0.794, p<0.0001$ & $r=0.412, p<0.0001$ \\
miR-30 & & & $r=0.786, p<0.0001$ & $r=0.329, p=0.0006$ \\
miR-192 & & & & $r=0.229, p=0.02$ \\
\hline
\end{tabular}

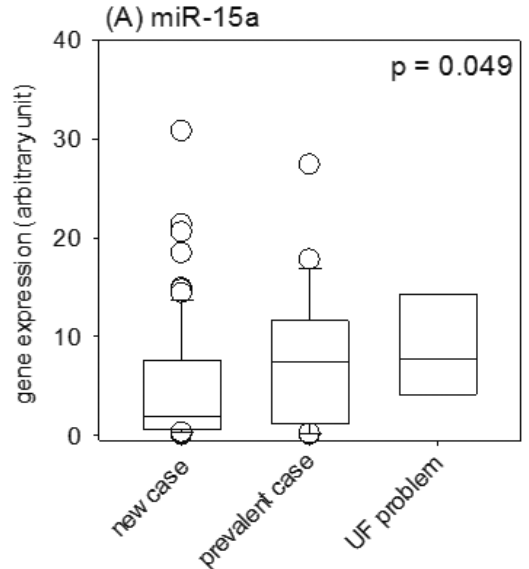

(D) $\mathrm{miR}-30$

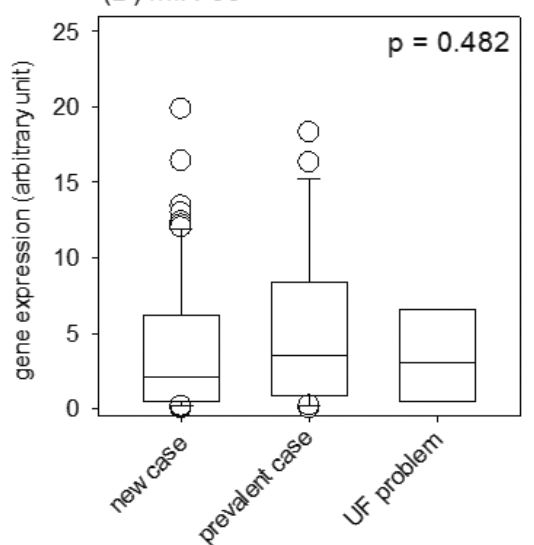

(B) miR-17

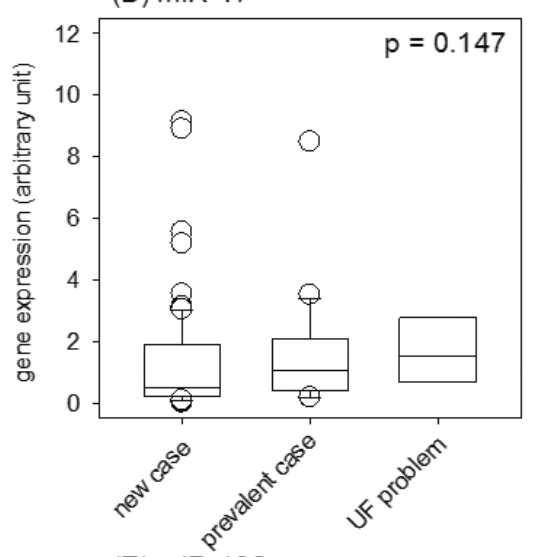

(E) $\operatorname{miR}-192$

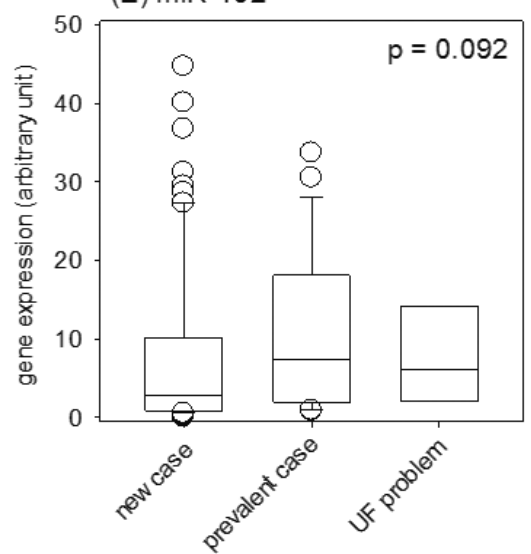

(C) miR-21

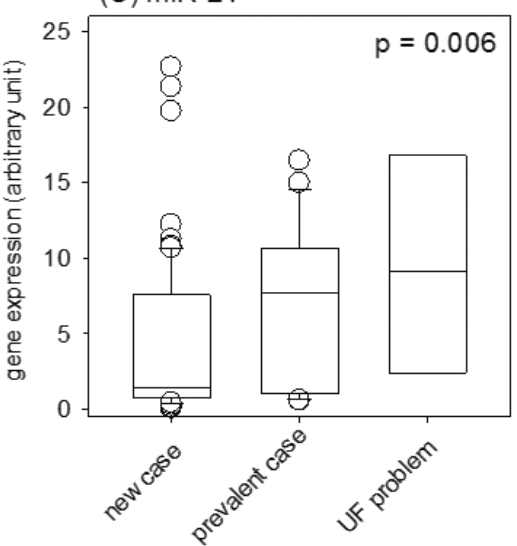

(F) $\operatorname{miR}-377$

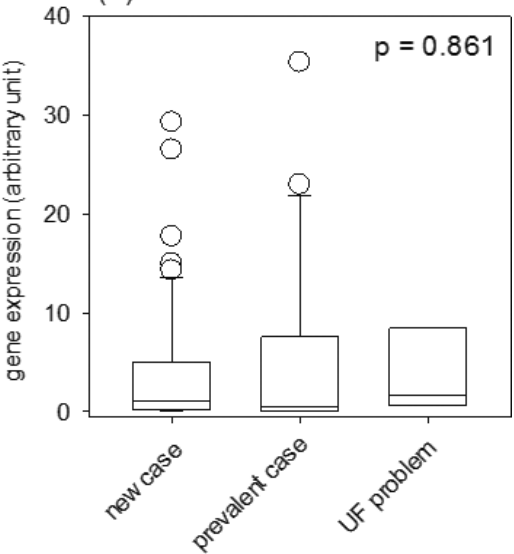

Fig. 1. Comparison of miRNA expression in peritoneal dialysis effluent between new PD patients, prevalent patients without ultrafiltration problem, and patients with ultrafiltration (UF) problem: (A) miR-15a; (B) miR-17; (C) miR-21; (D) miR-30; (E) miR-192; and (F) miR-377. Data are compared by Kruskal Wallis test.

sion of miR-216a and miR-217 was found in all samples. There was a close internal correlation between the PDE expressions of the other targets (Table 3). We noticed that PDE expression of miR-30 had a significant correlation with residual GFR $(r=-0.290, p=$ 0.010). PDE expression of other miRNA targets did not correlate with patients' age, duration on dialysis, Charlson comorbidity score, other baseline biochemical or dialysis adequacy parameters (details not shown).

PDE expression of individual miRNA targets are summarized Fig. 1. In essebce, the three groups had a different peritoneal expression of miR-15a (Kruskal Wallis test, $p=0.049)$, miR-21 ( $p=0.006)$, and miR$192(p=0.092)$, although the difference in miR-192 did not reach statistical significance. In contrast, PDE expression of miR-17, miR-30 and miR-377 were similar between new, prevalent, and UF falure cases.

\subsection{Relation with peritoneal transport}

Since there was no substantial difference in the peritoneal transport characteristics between new and preva- 

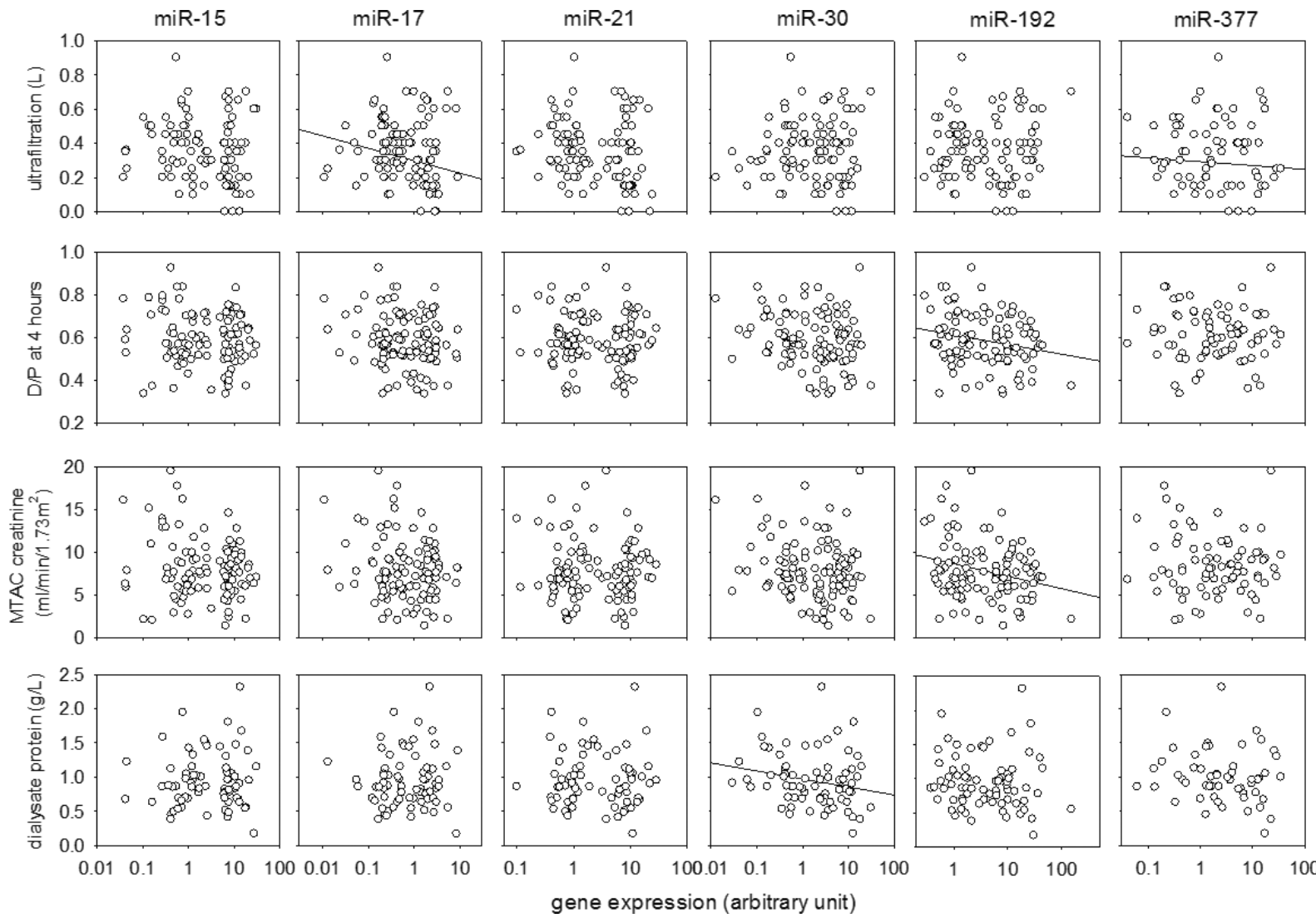

Fig. 2. Relation between miRNA expression in peritoneal dialysis effluent and peritoneal transport characteristics. Data are compared by Spearman's rank correlation coefficient.

lent PD patients, their data were pooled for the subsequent analysis. The relation between PDE miRNA expression and peritoneal transport characteristics is summarized in Fig. 2. There were modest but statistically significant inverse correlations between ultrafiltration volume and PDE expression of miR-17 $(r=-0.198$, $p=0.041)$ and miR-377 $(r=-0.201, p=0.041)$. There was an inverse correlations between $\mathrm{D} / \mathrm{P}$ at 4 hours and PDE expression of miR-192 $(r=-0.199$, $p=0.040)$; while MTAC creatinine correlated with PDE expression of miR-192 $(r=-0.191, p=0.049)$ and miR-377 ( $r=0.201, p=0.041)$. Dialysate protein concentration had significant correlation with PDE expression of miR-30 $(r=-0.247, p=0.035)$.

\subsection{Relation with change in peritoneal transport}

PET was repeated one year after the initial assessment in 7 randomly selected patients, and we explored the relation between baseline PDE miRNA expression and the change in peritoneal transport characteristics after one year. In short, there was a significant correlation between baseline PDE expression of miR-377 and change in ultrafiltration volume $(r=-0.852, p=$ 0.015) (Fig. 3). Amongst all miRNA targets that we examined, PDE expression of none of them correlated with the change in $\mathrm{D} / \mathrm{P}$ in 4 hour or MTAC creatinine over 12 months.

\section{Discussion}

In the study we found that the miR-15a, miR-17, miR-21, and miR-192 expression in PDE was substantially higher in prevalent patients (who either had suspected ultrafiltration problem or recently peritonitis) than new PD patients, while PDE expression of miR17, miR-192 and miR-377 inversely correlated with the peritoneal transport characteristics. Notably, the PDE level of many of the miRNA targets tested in this study had a close internal correlation, suggesting that their expressions are under a common control mechanism. Our results are consistent with previous studies in chron- 


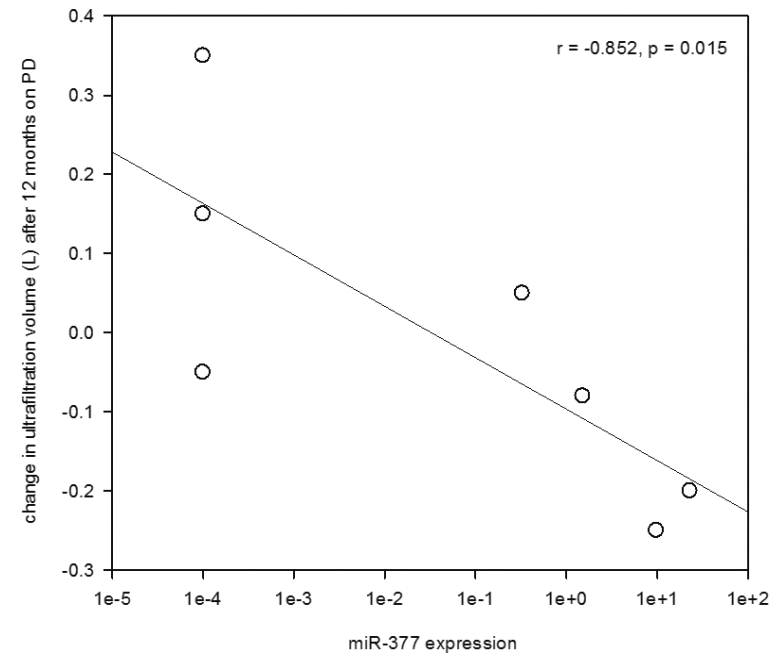

Fig. 3. Relation between miR-377 expression in peritoneal dialysis effluent and the change in ultrafiltration volume after 12 months on peritoneal dialysis.

ic kidney diseases, which generally indicated that the above miRNA species are probably related to renal fibrosis and epithelial-mesenchymal transition (EMT) of renal tubular cells [20]. For example, Lee et al. [21] found that miR-15a modulates the expression of the cell-cycle regulator $\mathrm{Cdc} 25 \mathrm{~A}$ and affects cystogenesis in a rat model of polycystic kidney disease. In experimental diabetic nephropathy, Zhang et al. [22] found that miR-21 prevents mesangial cell proliferation, Kato et al. [23] showed that miR-192 modulates transforming growth factor-beta (TGF- $\beta$ )-induced collagen expression via inhibition of E-box repressors, while Wang et al. [24] noted that miR-377 leads to increased fibronectin production in diabetic nephropathy. Taken together, our results indicate that, similar to renal fibrosis in chronic kidney diseases, these miRNA species may also be critical in the control of peritoneal transport and contribute to progressive peritoneal fibrosis. Our result may also suggest that these miRNA targets may have the potential of being developed as biomarkers of peritoneal failure, but the exact role in this aspect deserves further study.

It is important to realize that we quantified the miRNA levels in PDE sediments after centrifugation, which essentially represents intra-cellular miRNA. Although peritoneal membrane is permeable to miRNA, and circulating free miRNAs could gain entrance to the peritoneal cavity, these miRNAs should remain extracellular (i.e. present in dialysate supernatant after centrifugation) and are not detected in the present study. For this reason, we did not correct for the corresponding plasma miRNA levels, and the result should not be affected by a difference in the peritoneal permeability to large molecules.

Unfortunately, we did not observe any difference in ultrafiltration or transport parameters between the incident patients and those with ultrafiltration problem. The underlying reason for this intriguing finding remains obscure. It is possible that our sample size was small and the lack of difference may represent a type 2 statistical error. On the other hand, since ultrafiltration problem was defined clinically in our study (see $P a$ tients and Methods), it is possible that some of these patients might actually have gradual loss of residual renal function or suboptimal compliance to fluid restriction rather than a problem of peritoneal transport. It would have been ideal to compare prevalent PD patients with and without genuine ultrafiltration problems, but the group of prevalent patient in our study was too small for post hoc subgroup analysis.

Since most of the interesting targets we identified contributed to the process of EMT, our results indirectly support the notion that EMT is important in the determination of longitudinal change of peritoneal function. Our finding is consistent with the report of Yanez et al. [4], who reported that at the same time dialysis is initiated, peritoneal mesothelial cells underwent EMT, with a progressive loss of epithelial morphology and a decrease in the expression of cytokeratins and E-cadherin through an induction of the transcriptional repressor snail and up-regulation of expression of $\alpha 2$ integrin, and TGF- $\beta$ plays a critical role in this process. Recent studies in chronic kidney diseases suggest that miR-192 and miR-377 are critical in the process of TGF- $\beta$-induced fibrosis and EMT. Notably, TGF$\beta 1$ up-regulated miR-192 in rat tubular epithelial cells in vitro [25]. Over-expression of miR-192 amplified TGF- $\beta 1$-induced tubular collagen I expression, while inhibition of miR-192 blocked tubular collagen I expression in response to TGF- $\beta 1$ [25]. On the other hand, miR-377 was found to be up-regulated in cultured human and mouse mesangial cells by levels of glucose and TGF- $\beta$, and in animal models of type I diabetes [24]. In turn miR-377 reduced expression of serine/threonine protein kinase PAK1 and superoxide dismutase, which subsequently led to augmented fibronectin protein production [24]. In addition, miR377-mediated reduction in superoxide dismutase expression may result in increased oxidative stress that could also trigger fibrosis [24].

There are several limitations in our study. First, the choice of miRNA target for the study was diffi- 
cult. In our present study, we quantified miR-15a, miR17, miR-17-92, miR-21, miR-30, miR-192, miR-216a, miR-217, and miR-377 because previous studies in other organ systems suggest that they are involved in the process of EMT [20,26]. We believe further research should be performed by a hypothesis-free technology (for example, microarray) in order to explore all relevant miRNA species involved in peritoneal biology. In addition, since our sample size was relatively small, and the correlations between some of the miRNAs and peritoneal transport parameters were weak, our results need to be interpreted with caution.

Second, we used new PD patients as the comparator for prevalent cases with ultrafiltration problem, while, at least in theory, a better control group would be prevalent PD patients without ultrafiltration problem. In theory, if miRNA is a good marker of EMT in peritoneal membrane, they should have significant changes in patients on long term PD and evelop ultrafiltration problems, while EMT is unlikely to happen in new PD patients. In addition, any difference we found may therefore represent the effects of chronic exposure to a high glucose concentration. Unfortunately, we do not routinely test the peritoneal transport of prevalent PD patients without ultrafiltration problem and such data are not available.

In addition, we examined the total miRNA expression in dialysis effluent, and the cellular origin of the miRNA was not confirmed. However, our previous study showed that macrophage and mesothelial cells are the major cell types in the effluent of stable PD patients [27]. Since all patients in this study were free of peritonitis for over one month (see Methods), inflammatory cells should have little contribution to the miRNA. Further study is needed to confirm the relative contribution of individual miRNA species by each cell type. Similarly, our study is observational, and we did not prove the link between miRNA expression and peritoneal transport. Since the sample size was small for the longitudinal change in peritoneal transport, further studies of larger sample size and prospective follow up are needed to clarify the role of miRNA in this aspect.

In summary, we found that miRNA expression in PDE, including miR-15a, miR-17, miR-21, miR-30, miR-192, and miR-377, correlated with peritoneal transport characteristics in a various degree. Our result suggests that miRNA may play a role in the regulation of peritoneal membrane function.

\section{Conflict of interest}

All authors declare no conflict of interest.

\section{Acknowledgement}

This study was supported in part by the Hong Kong Society of Nephrology Research Grant, the Chinese University of Hong Kong (CUHK) research account 6901031 and the Richard Yu CUHK Peritoneal Dialysis Research Fund. Dr. J Chen was supported by the Stand Tall Programme of CUHK. The results presented in this paper have not been published previously in whole or part, except in abstract format. All authors declare no conflict of interest.

\section{References}

[1] Szeto CC, Wong TY, Leung CB, Wang AY, Law MC, Lui $\mathrm{SF}, \mathrm{Li} \mathrm{PK}$. Importance of dialysis adequacy in mortality and morbidity of Chinese CAPD patients. Kidney Int 2000; 58: 400-407.

[2] Kawaguchi Y, Hasegawa T, Nakayama M, Kubo H, Shigematu $\mathrm{T}$. Issues affecting the longevity of the continuous peritoneal dialysis therapy. Kidney Int Suppl 1997; 62: S105-107.

[3] Nagy J. Peritoneal membrane morphology and function. Kidney Int 1996 Suppl; 56: S2-S11.

[4] Yáñez-Mó M, Lara-Pezzi E, Selgas R, Ramírez-Huesca M, Domínguez-Jiménez C, Jiménez-Heffernan JA, Aguilera A, Sánchez-Tomero JA, Bajo MA, Alvarez V, Castro MA, del Peso G, Cirujeda A, Gamallo C, Sánchez-Madrid F, LópezCabrera M. Peritoneal dialysis and epithelial-to-mesenchymal transition of mesothelial cells. N Engl J Med 2003; 348: 403413.

[5] Williams JD, Craig KJ, Topley N, Von Ruhland C, Fallon M, Newman GR, Mackenzie RK, Williams GT. Morphologic changes in the peritoneal membrane of patients with renal disease. J Am Soc Nephrol 2002; 13: 470-479.

[6] Eulalio A, Huntzinger E, Izaurralde E. Getting to the root of miRNA-mediated gene silencing. Cell 2008; 132: 9-14.

[7] Ross JS, Carlson JA, Brock G. miRNA. the new gene silencer. Am J Clin Pathol 2007; 128: 830-836.

[8] He L, He X, Lowe SW, Hannon GJ. microRNAs join the p53 network-another piece in the tumour-suppression puzzle. Nat Rev Cancer 2007; 7: 819-822.

[9] Kuehbacher A, Urbich C, Dimmeler S. Targeting microRNA expression to regulate angiogenesis. Trends Pharmacol Sci 2008; 29: 12-15.

[10] Sassen S, Miska EA, Caldas C. MicroRNA: implications for cancer. Virchows Arch 2008; 452: 1-10.

[11] Korpal M, Lee ES, Hu G, Kang Y. The miR-200 family inhibits epithelial-mesenchymal transition and cancer cell migration by direct targeting of E-cadherin transcriptional repressors ZEB1 and ZEB2. J Biol Chem 2008; 283: 14910-14914.

[12] Gregory PA, Bert AG, Paterson EL, Barry SC, Tsykin A, Farshid G, Vadas MA, Khew-Goodall Y, Goodall GJ. The miR200 family and miR-205 regulate epithelial to mesenchymal transition by targeting ZEB1 and SIP1. Nat Cell Biol 2008; 10: 593-601.

[13] Burk U, Schubert J, Wellner U, Schmalhofer O, Vincan E, Spaderna S, Brabletz T. A reciprocal repression between ZEB1 and members of the miR-200 family promotes EMT and invasion in cancer cells. EMBO Rep 2008; 9: 582-589. 
[14] van Biesen W, Heimburger O, Krediet R, Rippe B, La Milia V, Covic A, Vanholder R. Evaluation of peritoneal membrane characteristics: clinical advice for prescription management by the ERBP working group. Nephrol Dial Transplant 2010; 25: 2052-2062.

[15] Mak TW, Cheung CK, Cheung CM, Leung CB, Lam CW, Lai KN. Interference of creatinine measurement in CAPD fluid was dependent on glucose and creatinine concentrations. Nephrol Dial Transplant 12: 184-186, 1997.

[16] Krediet RT, Boeschoten EW, Zuyderhoudt FMJ, Strackee J, Arisz L. Simple assessment of the efficacy of peritoneal transport in continuous ambulatory peritoneal dialysis patients. Blood Purification 1986; 4: 194-203.

[17] Szeto CC, Chow KM, Lam CW, Cheung R, Kwan BC, Chung $\mathrm{KY}$, Leung CB, Li PK. Peritoneal albumin excretion is a strong predictor of cardiovascular events in peritoneal dialysis patients: a prospective cohort study. Perit Dial Int 2005; 25: 445-452.

[18] Li B, Hartono C, Ding R, Sharma VK, Ramaswamy R, Qian B, Serur D, Mouradian J, Schwartz JE, Suthanthiran M. Noninvasive diagnosis of renal-allograft rejection by measurement of messenger RNA for perforin and granzyme B in urine. $\mathrm{N}$ Engl J of Med 2001; 344: 947-954.

[19] Szeto CC, Chan RW, Lai KB, Szeto CY, Chow KM, Li PK, Lai FM. Messenger RNA expression of target genes in the urinary sediment of patients with chronic kidney diseases. Nephrol Dial Transplant 2005; 20: 105-113.

[20] Li JY, Yong TY, Michael MZ, Gleadle JM. The role of microRNAs in kidney disease. Nephrology (Carlton) 2010; 15 : 599-608.
[21] Lee SO, Masyuk T, Splinter P, Banales JM, Masyuk A, Stroope A, Larusso N. MicroRNA15a modulates expression of the cell-cycle regulator Cdc25A and affects hepatic cystogenesis in a rat model of polycystic kidney disease. J Clin Invest 2008; 118: 3714-3724.

[22] Zhang Z, Peng H, Chen J, Chen X, Han F, Xu X, He X, Yan N. MicroRNA-21 protects from mesangial cell proliferation induced by diabetic nephropathy in $\mathrm{db} / \mathrm{db}$ mice. FEBS Lett 2009; 583: 2009-2014.

[23] Kato M, Zhang J, Wang M, Lanting L, Yuan H, Rossi JJ, Natarajan R. MicroRNA-192 in diabetic kidney glomeruli and its function in TGF-beta-induced collagen expression via inhibition of E-box repressors. Proc Natl Acad Sci USA 2007; 104: 3432-3437.

[24] Wang Q, Wang Y, Minto AW, Wang J, Shi Q, Li X, Quigg RJ. MicroRNA-377 is up-regulated and can lead to increased fibronectin production in diabetic nephropathy. FASEB J 2008; 22: 4126-4135.

[25] Chung AC, Huang XR, Meng X, Lan HY. miR-192 mediates TGF-beta/Smad3-driven renal fibrosis. J Am Soc Nephrol 2010; 21 : 1317-1325.

[26] Lorenzen JM, Haller H, Thum T. MicroRNAs as mediators and therapeutic targets in chronic kidney disease. Nat Rev Nephrol 2011; 7: 286-294.

[27] Lai KN, Lai KB, Lam CW, Chan TM, Li FK, Leung JC. Changes of cytokine profiles during peritonitis in patients on continuous ambulatory peritoneal dialysis. Am J Kidney Dis 2000; 35: 644-652. 


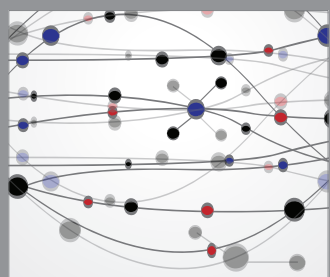

The Scientific World Journal
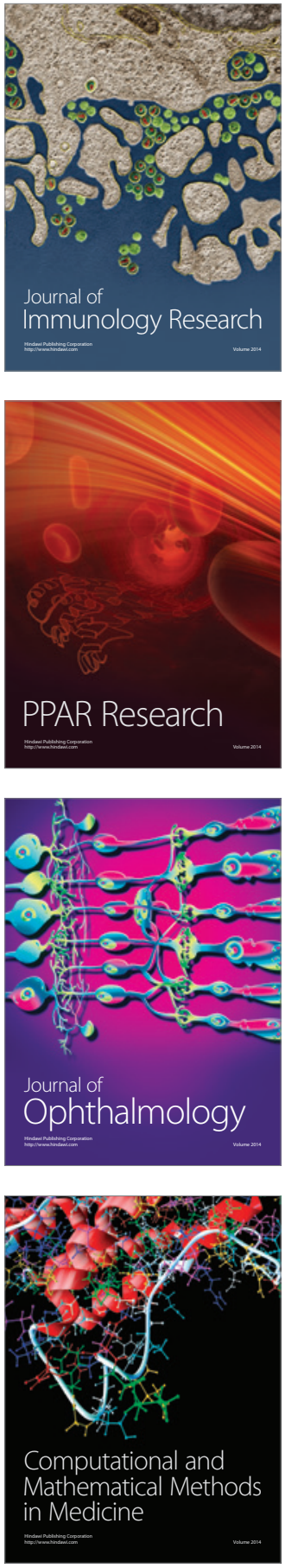

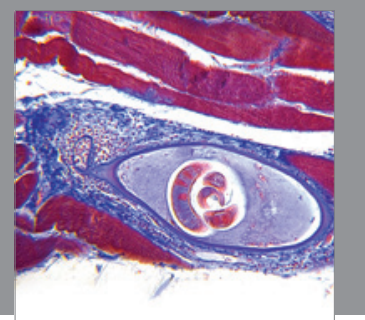

Gastroenterology

Research and Practice
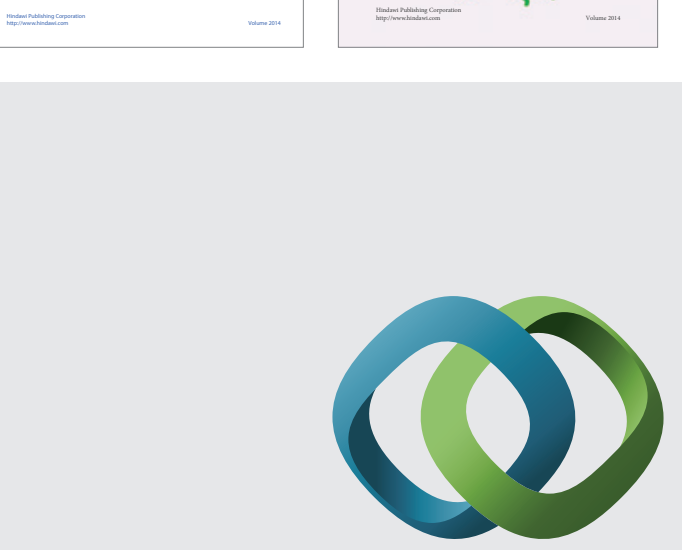

\section{Hindawi}

Submit your manuscripts at

http://www.hindawi.com
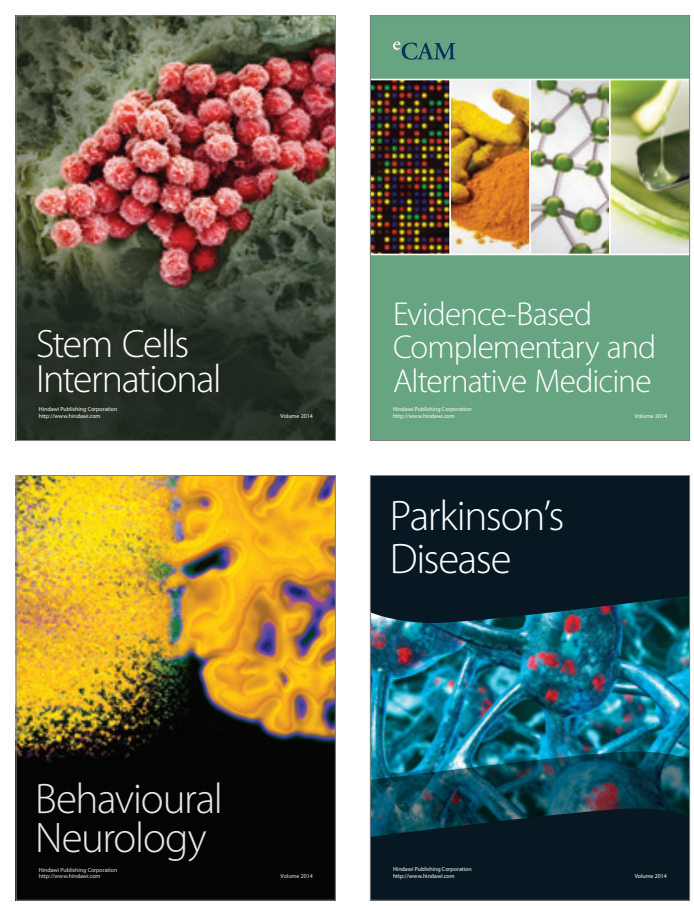

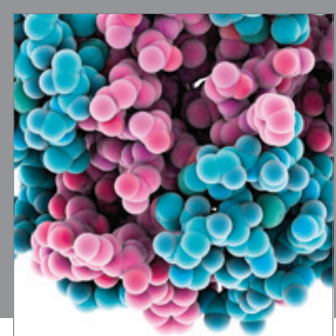

Journal of
Diabetes Research

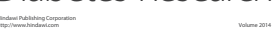

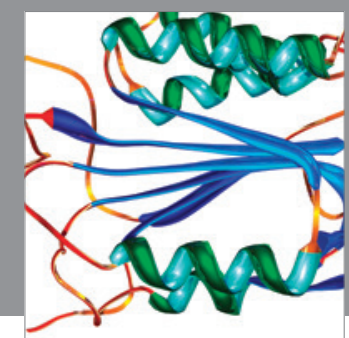

Disease Markers
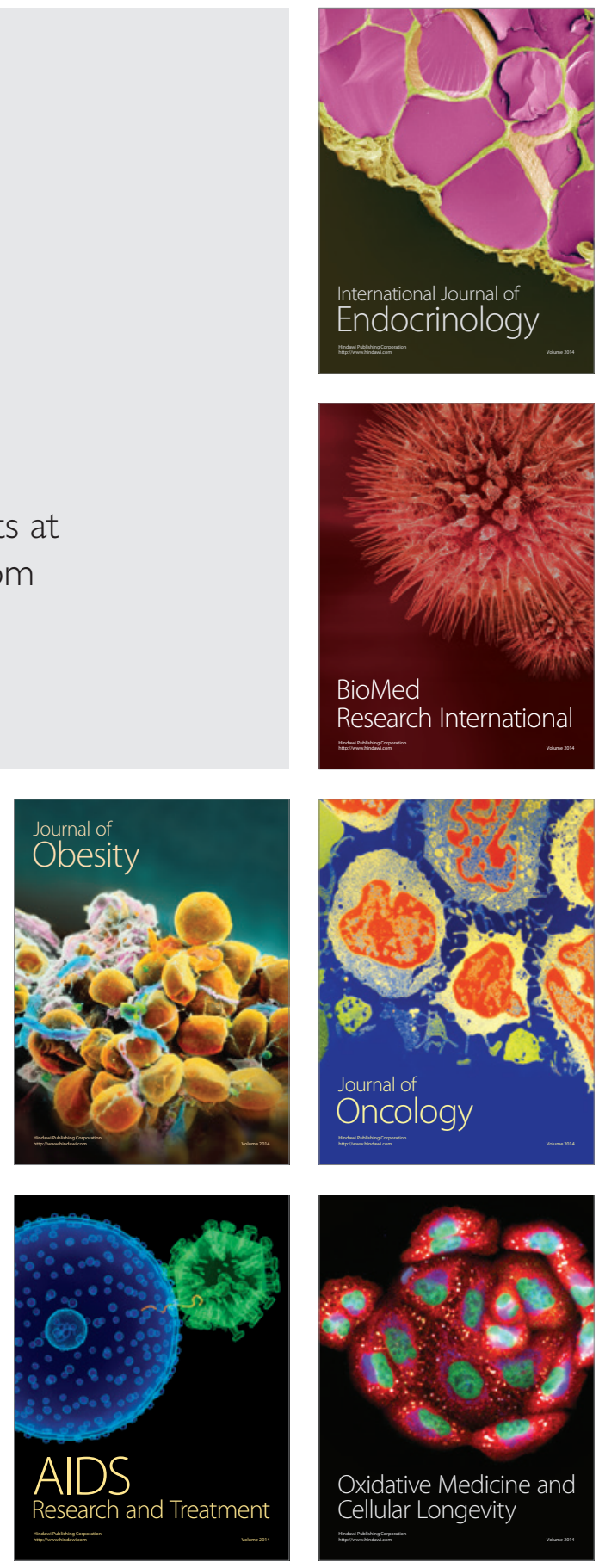